\title{
Waiting time paradox applied to transient times
}

\author{
P. J. Aston and P. K. Marriott \\ Department of Mathematical and Computing Sciences, University of Surrey, Guildford, Surrey GU2 5XH, United Kingdom
}

(Received 12 June 1997)

\begin{abstract}
Different methods for computing transient lengths in chaotic systems can give very different answers. This situation is resolved by the use of a waiting time paradox. [S1063-651X(98)05201-5]

PACS number(s): $05.45 .+\mathrm{b}$
\end{abstract}

\section{PROBLEM}

When working with chaotic systems, the average length of a transient phase before a particular event occurs is often required. Examples of this include the transient phase before a control mechanism can be activated $[1,2]$ or the length of a chaotic transient after a crisis has occurred [3]. In both cases, the transient occurs before iterates fall into a small region. For controlling chaos, this region is a target region, typically around a fixed or periodic point, in which control can then be applied. In the case of a crisis, the region is a small escape region.

There are different ways in which a transient phase can be computed numerically, with very different answers being obtained for the average length in some cases. We consider two methods for computing the average length of the transient which occurs before an iterate enters a particular small target or escape region.

(1) A large number of initial conditions is taken whose distribution is the same as the invariant probability distribution of the chaotic attractor. The lengths of the transient phases before entering the target region are then averaged.

(2) Let $p$ be the measure of the chaotic attractor contained in the target region. Then $p$ is also the probability of an iterate entering the target region. A standard calculation then gives that the average length of the transient phase is $1 / p$.

The second method is often used when deriving exponents in scaling laws for transient times $[2,3]$. To illustrate the different methods, we consider the Hénon map

$$
\begin{gathered}
x_{n+1}=1-1.4 x_{n}^{2}+y_{n}, \\
y_{n+1}=0.3 x_{n},
\end{gathered}
$$

which has a saddle fixed point at $\left(x^{*}, y^{*}\right)$ $=(0.63135,0.18941)$. When controlling chaos, the typical target region consists of a parallelogram centred on a fixed point with sides parallel to the stable and unstable manifolds of the fixed point. Such a target region is shown in Fig. 1.

The initial conditions are obtained for the first method by taking every 1000th iterate of a long orbit. Averaging over 10000 initial conditions gives an average transient length before entering the target region of $\tau_{1}=249.639$. For the second method, the probability $p$ is calculated as the number of iterates in the target region divided by the total number of iterates in a long orbit. Using an orbit of length $3 \times 10^{6}$ gives a value of the probability $p$ to be $p=7.987 \times 10^{-3}$, and thus an average transient length of $\tau_{2}=125.203$. Clearly $\tau_{2}$ is almost exactly one half of $\tau_{1}$.

\section{WAITING TIME PARADOX}

Consider the following classical paradox from probability theory. Suppose that a system follows a Poisson process in which a series of events, the arrivals, occur randomly in time such that the following two postulates hold: (A) The arrivals in the time interval $(t, t+h]$ are independent of the arrivals in the time interval $(0, t]$. This is the memoryless property of a Poisson process. (B) The probability of a single event occurring in a small time interval $(t, t+h]$ is $\tau h+o(h)$, while the probability of more than one event is $o(h)$. An alternative and equivalent characterization of the Poisson process is that the interarrival times (the times between consecutive events) are independently distributed with an exponential distribution. That is,

$$
P(T>t)=e^{-t / \tau}
$$

where $T$ is the interarrival time and $\tau$ is the average interarrival time.

There are two contradictory arguments for estimating the average time before the next arrival from a random starting time. Let the time for the next arrival starting from time $t$ be $W_{t}$. The two arguments are then as follows: (i) The average time between arrivals is $\tau$. Hence by arriving at random, symmetry tell us that the average arrival time is $\frac{1}{2} \tau$. (ii) The

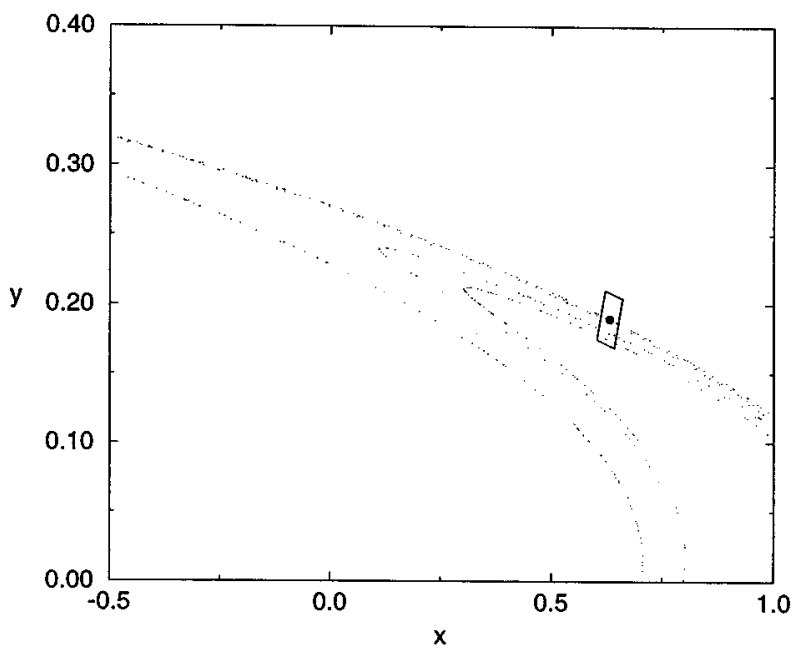

FIG. 1. Target region around a fixed point. 
Poisson process has no memory hence the expected waiting time should be independent of $t_{0}$ the initial time. Therefore,

$$
E\left(W_{t}\right)=E\left(W_{t_{0}}\right)=\tau \text {. }
$$

Both arguments are very plausible. The problem is understood by noting that there is a difference between averaging over all gaps, and averaging over gaps chosen by a random initial time. In choosing a random arrival time you are much more likely to arrive in the middle of a long gap than a short one. In fact, as a simple calculation shows, the average length of a gap chosen at random is $2 \tau$ (see Ref. [4]). Hence the paradox is resolved.

\section{APPLICATION TO CALCULATION OF TRANSIENTS}

To apply the above theory to the chaotic system we consider discrete time which we partition into blocks of $m$ iterations such that the position after $(k+1) m$ iterations is (approximately) independent of that after $\mathrm{km}$ iterations. Let $T$ be the number of iterations until the first entry into the target region and define

$$
T_{m}=\inf \{k \mid k m>T\} .
$$

By the independence property we can apply a Bernoulli model to the distribution of $T_{m}$. Hence if $p_{m}=P\left(T_{m}=1\right)$ then,

$$
P\left(T_{m}>k\right)=\left(1-p_{m}\right)^{k}
$$

and

$$
E\left[T_{m}\right]=\frac{1}{p_{m}}
$$

We also have the approximation that

$$
E[T]=m E\left[T_{m}\right]=\mu,
$$

say. Combining these gives

$$
\begin{aligned}
P\left(T_{m}>k\right) & =\left(1-p_{m}\right)^{k}=(1-m / \mu)^{k} \approx(1-m / \mu)^{t / m} \\
& =\left[(1-m / \mu)^{\mu / m}\right]^{t / \mu} .
\end{aligned}
$$

Hence, moving back from $T_{m}$ to $T$ and appealing to the smoothing properties of probability distributions, we have the approximation

$$
P(T>t)=e^{-t / \mu},
$$

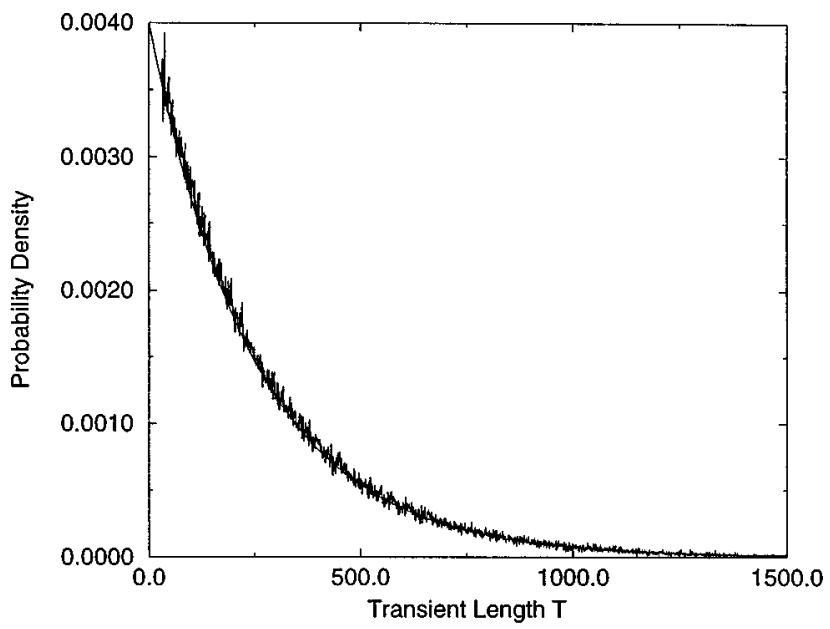

FIG. 2. Distribution of transient lengths.

provided that $m / \mu$ is small. In other words if the average number of iterations needed between hitting the target region is large relative to the memory of the process then the times between hits are well approximated by an exponential distribution for sufficiently long transient lengths. Hence the Poisson process model is appropriate.

The distribution of transient lengths for the example in Sec. I is shown in Fig. 2. There is considerable variation for small values of $T$, and so these have not been included. The curve $e^{-T / \tau_{1} / \tau_{1}}$ is also shown, where $\tau_{1}$ is the average obtained earlier, and clearly there is good agreement. This type of distribution has been observed previously in the calculation of transients [3].

This approach gives a direct interpretation of the results of Sec. I in terms of the waiting time paradox. The second method averages equally over all interarrival times and corresponds to argument (A), so that $\tau_{2}=\mu / 2$, which is half the average interarrival time, while the first method finds the average waiting time given a random starting time which corresponds to argument (B). This weights the average toward the longer waiting times giving $\tau_{1}=\mu$.

Finally, we note that in many cases, scaling laws with respect to a parameter are derived for transients, and these are then tested numerically $[2,3]$. Clearly the scaling law will be the same which ever method is used for calculating the transients. However, if a comparison of absolute lengths of transients is required, it is clearly essential to use the same method in both cases to obtain a true comparison.
[1] P. J. Aston and C. M. Bird, Chaos Solitons Fractals 8, 1413 (1997).

[2] E. Ott, C. Grebogi, and J. A. Yorke, Phys. Rev. Lett. 64, 1196 (1990).
[3] C. Grebogi, E. Ott, and J. A. Yorke, Phys. Rev. Lett. 57, 1284 (1986).

[4] W. Feller, An Introduction to Probability Theory and Its Applications (Wiley, London, 1966). 